\title{
SATISFAÇÃO EM ENFERMAGEM: PERSPETIVA DO ENFERMEIRO VERSUS PERSPETIVA DO UTENTE
}

\author{
Eugénia Maria Garcia Jorge Anes \\ Escola Superior de saúde de Bragança, IPB \\ eugenia@ipb.pt \\ Carina Alexandra Salvador Ferreira \\ Unidade Local de Saúde do Nordeste - Unidade de Mirandela
}

Fecha de Recepción: 15 Marzo 2018

Fecha de Admisión: 10 Abril 2018

\begin{abstract}
RESUMO
A dupla vertente da satisfação dos utentes e da satisfação profissional dos enfermeiros constitui um indicador de resultado, válido na avaliação da qualidade dos serviços. Temos como objetivo avaliar a satisfação dos enfermeiros e a satisfação dos utentes com os cuidados de enfermagem, em meio hospitalar. Foram utilizadas: a escala EAST-Enf, a escala SUCEH21 e questões de caracterização. Desenvolveu-se um estudo transversal, descritivo e exploratório, quantitativo, numa amostra de 124 enfermeiros e 462 utentes no norte de Portugal. A amostra dos profissionais é predominantemente feminina (82.3\%), com média de idade a rondar os 42 anos. Dos 462 utentes, a maioria é do sexo feminino (53.2\%), com idade média de 57.47 anos. Os enfermeiros mais satisfeitos profissionalmente são os mais jovens $(84,13)$, com menor grau académico $(74,80)$, com tempo de serviço inferior a 10 anos $(78,59)$, que trabalham na unidade de $\operatorname{AVC}(77,10)$, e aqueles que exercem funções em serviços com liderança democrática (76.6\%). Relativamente aos utentes, os mais satisfeitos com os cuidados de enfermagem são as mulheres $(2,87)$, os mais idosos $(2,87)$, viúvos $(2,59)$, reformados $(2,56)$, internados na unidade de AVC (3). Na satisfação dos enfermeiros, destacam-se como variáveis determinantes, a idade dos enfermeiros e o tempo de serviço. A satisfação dos utentes com os cuidados de enfermagem destaca-se o sexo, idade, estado civil, situação laboral, profissão e serviço de internamento. De forma global, verificou-se uma relação direta entre a satisfação dos profissionais e a satisfação dos utentes. A identificação dessas relações é muito importante nas organizações de saúde, constituindo uma ferramenta sólida na tomada de decisão e, portanto, na prestação de melhores cuidados de saúde.

Palavras-chave: satisfação no emprego; enfermeiros; enfermagem; cuidados de enfermagem; satisfação do utente
\end{abstract}




\section{SATISFAÇÃO EM ENFERMAGEM: PERSPETIVA DO ENFERMEIRO VERSUS PERSPETIVA DO UTENTE}

\section{ABSTRACT}

Satisfaction in nursing: perspective of the nurse versus the perspective of the patient.

The double aspect of the satisfaction of the users and the professional satisfaction of the nurses is an indicator of result, valid in the evaluation of the quality of the services. We aim to evaluate the satisfaction of nurses and the satisfaction of patients with nursing care, in a hospital setting. The EAST-Enf scale, the SUCEH21 scale and characterization questions were used. A cross-sectional, descriptive and exploratory, quantitative study was developed in a sample of 124 nurses and 462 patients in northern Portugal. The sample of professionals is predominantly female (82.3\%), with an average age of around 42 years. Of the 462 users, the majority are female (53.2\%), with a mean age of 57.47 years. The most professionally satisfied nurses are the youngest $(84,13)$, with a lower academic rank $(74,80)$, with a service period of less than 10 years $(78,59)$, who work in the stroke unit $(77,10)$, and those who work in services with democratic leadership (76.6). Among the users, the most satisfied with nursing care are women $(2,87)$, the elderly $(2,87)$, widowers $(2,59)$, retired $(2,56)$, hospitalised in the unit of stroke (3). In nurses' satisfaction, the determinants, the age of the nurses and the service time stand out. The satisfaction of the users with the nursing care stands out the sex, age, marital status, labor situation, profession and internment service. Overall, there was a direct relationship between the satisfaction of the professionals and the satisfaction of the users. The identification of these relationships is very important in health organizations, constituting a solid tool in decision making and, therefore, in providing better health care.

Keywords: job satisfaction; nurses; nursing; nursing care; patient satisfaction

\section{INTRODUÇÃO}

A saúde desempenha um papel fundamental no desenvolvimento dos países no campo económico-financeiro, social e cultural. Nas sociedades desenvolvidas tornou-se um tema dominante nos discursos social e político, pelo que as organizações de saúde devem estar atentas para se manterem e serem competitivas no mercado global demonstrando eficácia e eficiência dos seus serviços.

Um indicador de resultado, válido na avaliação da qualidade dos serviços, é a satisfação, o qual tem vindo a ocupar um lugar importante e permanente, quer por parte das instituições de saúde, quer por parte dos governantes, sendo um objetivo major na vida dos indivíduos. Pode ser entendida como uma ferramenta importante de gestão de recursos humanos, contribuindo para o bom funcionamento do trabalhador e da organização, com consequências na qualidade de vida laboral (Cañon Buitrago \& Galeano Martinez, 2011).

A satisfação pode e deve ser vista segundo dois prismas, tendo em atenção a sua inter-relação, ou seja, a satisfação dos profissionais de saúde, aqui de forma específica a satisfação dos enfermeiros e a satisfação dos utentes. A satisfação em enfermagem é uma área complexa e de difícil avaliação.

A informação resultante da investigação científica permite-nos identificar os fatores determinantes da satisfação em enfermagem, possibilitando a tomada de decisão no sentido de a melhorar, contribuindo deste modo para a melhoria contínua da qualidade de vida dos indivíduos e da qualidade dos cuidados prestados.

A satisfação profissional é um constructo que visa dar resposta a um estado emocional ou a uma atitude face ao trabalho e às experiências em contexto de trabalho, que na literatura científica tem dificuldade em definir de forma clara e consensualmente aceite entre os investigadores.

A satisfação dos profissionais de saúde é, de acordo com a Lei de Bases da Saúde, um dos quatro critérios de avaliação periódica do serviço nacional de saúde (SNS), em conjunto com a satisfação dos utentes, a qualidade dos cuidados e a eficiente utilização dos recursos, do ponto de vista 
do custo/benefício (Decreto-lei [DL] 꾸 48/90, de 24 de agosto, revogada pela Lei n.. 27/2002, de 8 de novembro).

A satisfação profissional pode ser entendida como "um processo que resulta da complexa e dinâmica interação das condições gerais da vida, das relações do trabalho, do processo de trabalho e do controle que os próprios trabalhadores possuem sobre as suas condições de vida e de trabaIho" (Marqueze \& Moreno, 2005, p.77). Ou como um estado estacionário de relaxamento resultante da perceção de cumprimento de necessidades face ao trabalho (Cunha, Rego, Cunha, CabralCardoso \& Neves, 2006).

A temática da satisfação dos utentes tem sido nos últimos anos uma preocupação crescente para os gestores da área da saúde. Esta filosofia de gestão caracteriza-se essencialmente pela sua focalização nos utentes, valorizando e respeitando cada vez mais a opinião destes, no sentido de "monitorizar a qualidade dos serviços de saúde e de avaliar a eficácia das medidas corretivas a ser implementadas" (Mendes, Mantonavi, Gemito, \& Lopes, 2013, p.18). Numa realidade em que cada vez se fala mais na gestão e prática de cuidados de qualidade, colocar o utente em primeiro lugar é uma das filosofias da qualidade.

Considerando a satisfação como um indicador da qualidade de cuidados de saúde, 0 utente vai proceder à avaliação dos cuidados que the foram prestados com base nas suas necessidades e com base nas experiências prévias pessoais e de outros e ainda, da eficácia da comunicação.

0 termo satisfação provém etimologicamente do latim satisfactione. São variadíssimos os conceitos de satisfação do utente, mas é unânime a sua relação com as expectativas dos utentes face à qualidade percebida dos serviços. Ferreira $(2014$, p. 6) apresenta a fórmula elucidativa e explicativa da satisfação de McIntyre e Silva (1999), sendo"a satisfação dos utentes (S) com os cuidados de saúde 0 resultado da diferença entre as expetativas do utente em relação aos cuidados $(E)$ e a sua perceção dos cuidados realmente recebidos (P), ou seja S=E-P”.

A Estratégia Nacional para a Qualidade na Saúde 2009 -2014, aprovada pelo Despacho Ministerial n.․ 14223/2009, estabeleceu, como uma das áreas de atuação da Direção Geral da Saúde através do Departamento da Qualidade na Saúde, "a implementação de sistemas de monitorização periódica do grau de satisfação dos utilizadores do sistema de saúde" (Diário da República, 2. série n. -120 , de 24 de junho de 2009, pag, 24669).

Posteriormente, a Portaria $\mathrm{n} .-159 / 2012$, de 22 de maio, veio definir as competências específicas do Departamento da Qualidade na Saúde, estabelecendo a necessidade de "Gerir os sistemas de monitorização e perceção da qualidade dos serviços pelos utentes (...), e promover a avaliação sistemática da satisfação" (Diário da República, 1. a série, n.ำ99, p. 2673).

0 utente não deve assumir a postura de mero espectador no que se refere aos serviços e cuidados de saúde que lhe são oferecidos (Mendes et al., 2013). 0 utente é 0 elemento chave para a avaliação dos cuidados prestados, bem como para a melhoria contínua da qualidade. A voz do utente constitui fonte de referência na tradução da sua satisfação.

\section{OBJETIVO}

0 presente estudo tem como objetivo de investigação, avaliar a satisfação dos enfermeiros que exercem funções em meio hospitalar e a satisfação dos utentes face aos cuidados de enfermagem.

\section{METODOLOGIA}

Desenvolveu-se um estudo transversal, descritivo e exploratório, quantitativo, numa amostra de 124 enfermeiros e 462 utentes de uma unidade local de saúde do norte de Portugal. Foi fixado um erro amostral inicial de 5\%, recorrendo à fórmula de Oliveira e Grácio (2005). 


\section{SATISFAÇÃO EM ENFERMAGEM: PERSPETIVA dO ENFERMEIRO VERSUS PERSPETIVA DO UTENTE}

0 instrumento de colheita de dados é composto pelas escalas EAST-Enf (enfermeiros), a escala SUCEH21 (utentes) e questões de caracterização (sociodemográficas e clínicas).

A colheita de dados decorreu em 2016, foram observados os procedimentos éticos de acordo com 0 tratado de Helsínquia.

\section{RESULTADOS}

A amostra dos profissionais de enfermagem $(n=124)$ é predominantemente feminina $(82.3 \%)$, com média de idade a rondar os 42 anos. Maioritariamente casados ou em união de facto $(74.8 \%)$. Quanto à habilitação profissional, a maioria possuem licenciatura (46\%), seguindo com $31.5 \%$ enfermeiros com especialidade. Na categoria profissional contabilizaram-se $41.1 \%$ como enfermeiros e $40.3 \%$ como enfermeiros graduados. Quanto ao tempo de serviço, a média é de 17,1 anos. A maioria dos enfermeiros exerce funções na unidade hospitalar C (58.1\%), no serviço de ortopedia $(11.3 \%)$, trabalha por turnos $(82.3 \%)$, possui contrato de trabalho em funções públicas $(59.7 \%)$ e, como remuneração mensal, $46.8 \%$ aufere entre 1000 e 1200 euros.

Dos 462 utentes, a maioria é do sexo feminino (53.2\%), com idade média de 57.47 anos, casada ou vive em união de facto (57.6\%), reside em meio urbano (54.8\%), 50.2\% dos utentes possuem habilitações académicas inferiores ao ensino básico, $45 \%$ está no ativo e a maioria está internada na unidade hospitalar C (55\%), no serviço de medicina (24.9\%) e o motivo pelo qual a maioria dos utentes estava internada era devido a problemas do sistema digestivo (17.5\%).

No quadro 1 podem observar-se as diferenças nos níveis de satisfação dos enfermeiros. Não existem diferenças significativas nos níveis de satisfação dos enfermeiros em relação ao sexo, estado civil, habilitação profissional, categoria profissional, tipo de horário e regime contratual. Verificaram-se diferenças significativas em relação à idade, os mais satisfeitos profissionalmente são os mais jovens $(84,13)$, com menor grau académico $(74,80)$, com tempo de serviço inferior a 10 anos $(78,59)$, os que trabalham no serviço serviço de AVC $(77,10)$ e aqueles que exercem funções em serviços com liderança democrática $(75,62)$.

Quadro 1

Satisfação no trabalho para enfermeiros: EAST-Enf

\begin{tabular}{lcc}
\hline \multicolumn{1}{c}{ EAST-Enf } & Estatística teste & Valor prova \\
\hline Sexo & $\mathrm{t}=0,375$ & 0.708 \\
\hline Idade & $4.999^{\mathrm{a}}$ & $0.003^{*}$ \\
\hline Estado civil & $4.610^{\mathrm{a}}$ & 0.203 \\
\hline Grau académico & $\mathrm{t}=2.064$ & $0.041^{*}$ \\
\hline $\begin{array}{l}\text { Habilitação } \\
\text { profissional }\end{array}$ & $1.023^{\mathrm{a}}$ & 0.385 \\
\hline Categoria profissional & $0.760^{\mathrm{a}}$ & 0.470 \\
\hline Tempo de serviço & $6.881^{\mathrm{a}}$ & $0.032^{*}$ \\
\hline Serviço & $10.676^{\mathrm{a}}$ & 0,015 \\
\hline Regime contratual & $\mathrm{t}=0.262^{2}$ & 0.794 \\
\hline Tipo de liderança & $13.337^{\mathrm{a}}$ & $0.001^{*}$ \\
\hline Nota ${ }^{*}$ Significativo; ${ }^{\mathrm{a}}$ Teste Kruskal-Wallis; $\mathrm{t}=$ teste $\mathrm{T}-\mathrm{Student}$
\end{tabular}


Relativamente aos utentes (Quadro 2), existem diferenças estatisticamente significativas em relação ao sexo, sendo os mais satisfeitos com os cuidados de enfermagem são as mulheres $(2,87)$; a idade, em que o nível de satisfação é superior nos mais idosos (2,87); o estado civil, sendo os viúvos $(2,59)$ aqueles que apresentam melhores pontuações; a situação profissional, apresentando valores de satisfação superiores nos reformados $(2,56)$; e o serviço de internamento, sendo a unidade de AVC (3) que apresenta melhores pontuações.

Quadro 2

Satisfação dos utentes: SUCEH21

\begin{tabular}{lcccccc}
\hline & $\begin{array}{c}\text { Eficácia } \\
\text { comunicaç } \\
\text { ão }\end{array}$ & $\begin{array}{c}\text { Utilidade } \\
\text { informaç } \\
\text { ão }\end{array}$ & $\begin{array}{c}\text { Qualidade } \\
\text { atendiment } \\
\text { o }\end{array}$ & $\begin{array}{c}\text { Prontidã } \\
\text { o } \\
\text { assistênci } \\
\text { a }\end{array}$ & $\begin{array}{c}\text { Manutenção } \\
\text { ambiente } \\
\text { terapêutico }\end{array}$ & $\begin{array}{c}\text { Promoção } \\
\text { continuidade } \\
\text { cuidados }\end{array}$ \\
\hline Sexo & 0.471 & 0.414 & $0.048^{*}$ & 0.470 & 0.238 & $0.000^{*}$ \\
Idade & 0.337 & $0.000^{*}$ & $0.012^{*}$ & 0.229 & 0.150 & $0.000^{*}$ \\
Estado civil & 0.383 & $0.000^{*}$ & 0.077 & 0.217 & 0.691 & $0.000^{*}$ \\
Situação laboral & 0.904 & $0.001^{*}$ & 0.082 & 0.251 & 0.521 & $<0.001^{*}$ \\
Unidade hospitalar & $0.022^{*}$ & $0.000^{*}$ & $0.027^{*}$ & $0.000^{*}$ & $0.001 *$ & $0.000^{*}$ \\
Serviço & $0.033^{*}$ & $<0.001^{*}$ & $0.007^{*}$ & $<0.001^{*}$ & 0.068 & $<0.001^{*}$ \\
\hline
\end{tabular}

Nota.* Significativo

Globalmente, e relativamente ao serviço de internamento, verificou-se uma relação direta entre a satisfação dos profissionais e a satisfação dos utentes, encontrando-se níveis mais elevados tanto para os profissionais como nos utentes no serviço de AVC.

\section{DISCUSSÃO}

Na satisfação dos enfermeiros, destacam-se como variáveis determinantes, a idade dos enfermeiros, o grau académico, o tempo de serviço, o tipo de serviço e o tipo de liderança utilizado. Palha (2013) e Martinho (2015) corrobora os presentes resultados, afirmando que não existem diferenças entre enfermeiros e enfermeiras. Contrariamente aos resultados aqui encontrados, Maia (2012) e Palha (2013), concluíram que a satisfação global no trabalho aumenta com 0 aumento da idade.

Na satisfação dos utentes com os cuidados de enfermagem destaca-se 0 sexo, idade, estado civil, situação laboral, e o serviço de internamento. Ribeiro em 2003, Mota, Amorim, Trigueiro, Flores e Leuschner em 2010, Silva em 2011 e Ferreira em 2014 não encontraram diferenças em relação ao sexo. Franco e Florentim (2006), Raffi, Hajinezhad e Haghani (2008), Silva (2010) e Ferreira (2014) vem corroborar a presente investigação no que respeita à idade. Relativamente ao estado civil Ribeiro (2003), Oliveira e Guirardello (2006), Silva (2010) e Ferreira (2014) também corroboram os presentes resultados.

De forma global, e relativamente ao serviço de internamento, verificou-se uma relação direta entre a satisfação dos profissionais e a satisfação dos utentes.

\section{CONCLUSÃO}

Sendo a satisfação do utente um indicador da qualidade dos cuidados prestados e a satisfação 
do profissional determinante desta mesma qualidade, torna-se ide fulcros relevância a identificação dessas relações. Pelo que, nas organizações de saúde, constitui uma ferramenta sólida na tomada de decisão e, portanto, indispensável à prestação de melhores cuidados de saúde.

\section{REFERÊNCIAS BIBLIOGRÁFICAS}

Cañón Buitrago, S. C., \& Galeano Martinez, G. (2011). Factores laborales psicosociales y calidad de vida laboral de los trabajadores de la salud de ASSBASALUD ESSE Manizales (Colombia). Archivos de Medicina, 11(2), 114-126. Recuperado de http://www.redalyc.org /articulo.oa?id=273821489004

Lei no 27/2002, de 8 de novembro. Aprova o novo regime jurídico da gestão hospitalar e procede à primeira alteração à Lei n.o 48/90, de 24 de agosto. Diário da República, 258. Série I-

A.Cunha, M. P., Rego, A., Cunha, R. C., Cabral-Cardoso, C., \& Neves, P. (2006). Manual de comportamento organizacional e gestão (5a ed. rev. e atual.). Lisboa: Editora RH.

Marqueze, E. C., \& Moreno, C. R. C. (2005). Satisfação no trabalho: Uma breve revisão. Revista Brasileira de Saúde Ocupacional, 30(112), 69-79. Recuperado de http://www.scielo.br/pdf/rbso/v30n112/07.pdfMendes, F., Mantonavi,

M. F., Gemito, M. L., \& Lopes, M. J. (2013). A satisfação dos utentes com os cuidados de saúde primários. Revista de Enfermagem Referência, 9 (Série 3), 17-25.

Ferreira, C. A. S. (2014). Satisfação dos utentes da Unidade Local de Saúde do Nordeste face aos cuidados de enfermagem. Dissertação de mestrado não publicada. Instituto Politécnico de Bragança. Recuperado de https://bibliotecadigital.ipb. pt/bitstream/10198/9785/1 /Carina\%20Salvador\%20Ferreir a.pdf

Despacho n.o 14223/2009, de 24 de junho. Procede à aprovação da estratégia nacional para a qualidade na saúde. Diário da República, 120. Série II.

Portaria n.o 159/2012, de 22 de maio. Fixa a estrutura nuclear da Direção-Geral da Saúde. Diário da República, 99. Série I.

Mendes, F., Mantonavi, M. F., Gemito, M. L., \& Lopes, M. J. (2013). A satisfação dos utentes com os cuidados de saúde primários. Revista de Enfermagem Referência, 9 (Série 3), 17-25

Palha, M. F. (2013). Satisfação profissional dos enfermeiros em unidades de cuidados continuados integrados. Dissertação de mestrado não publicada. Instituto Politécnico do Porto. Recuperado de http://hdl.handle.net/10400.22/8952

Martinho, J. M. (2015). Satisfação profissional dos enfermeiros especialistas em enfermagem de reabilitação. Dissertação de mestrado não publicada. Instituto Politécnico de Viseu. Recuperado de http://hdl.handle.net/10400.19/3229

Maia, N. D. 0. (2012). Satisfação em enfermagem: Comparação entre sector público e privado. Dissertação de mestrado não publicada. Universidade de Aveiro. Recuperado de http://hdl.handle.net/10773/8792

Mota, S., Amorim, M. M., Trigueiro, M. J., Flores, C., \& Leuschner, A. (2010). Avaliação da satisfação e importância atribuída aos serviços prestados na área da saúde mental. Porto: Escola Superior de Tecnologia Saúde, Instituto Politécnico do Porto. Recuperado de http://recipp.ipp.pt/bitstream/10400.22/1319/1/COM_SMota_2010.pdf

Ribeiro, A. (2003). Satisfação dos utentes com os cuidados de enfermagem: Construção e validação de um instrumento de medida. Dissertação para concurso de provas públicas para professor coordenador não publicada. Escola Superior de Enfermagem São João, Porto.

Silva, M. C. C. (2011). Satisfação do doente cardíaco com os cuidados de enfermagem. Relatório final de mestrado não publicado. Escola Superior de Saúde, Instituto Politécnico de Viseu. 
Franco, M. J. B., \& Florentim, R. J. S. (2006). A satisfação dos utentes em serviços de saúde: Um estudo exploratório sobre o departamento de psiquiatria e saúde mental do Centro Hospitalar da Beira. Revista Portuguesa de Saúde Pública, 24(1), 21-35. 
